\title{
Essay Classifying science and technology: two problems with the UNESCO system
}

\author{
JESÚS MARTÍNEZ-FRÍAS and DAVID HOCHBERG \\ Centre for Astrobiology, CSIC/INTA, 28850 Torrejón de Ardoz, Madrid, Spain
}

Systems of classifying knowledge are of key importance because they can provide a kind of spatiotemporal 'snapshot' of science, of the sphere of ideas, of the culture of a given period. But such snapshots are, inevitably, perishable. In 1966, UNESCO assigned itself the task of developing an international standard system for classifying science and technology. ${ }^{1}$ In this it eventually succeeded, although it was not until 1988 that the now well known International Standard Nomenclature for Fields of Science and Technology was formally proposed. ${ }^{2}$ Despite the fact that this nomenclature was initially intended as a system for classification of research papers and doctoral dissertations, nowadays the UNESCO system has become an essential international guide for many organisations and research institutions, which use it for a whole variety of purposes, for example for definition of research guidelines, cataloguing thematic projects, advertising research positions with public bodies, categorising scientists for subsequent scientific evaluations, and so on.

In essence, the UNESCO system has three versions, classifying science and technology into fields (two-digit code), disciplines (four-digit code) and subdisciplines (six-digit code). The six-digit codes are probably the best known and most widely used. Two specific and significant classification problems exist with the system, however, the first in relation to misleading assignments of certain scientific disciplines and subdisciplines, the second to do with the necessity of updating categories as emerging disciplines and subdisciplines become established. These problems are well illustrated by the cases of planetary geology and astrobiology respectively.

\section{MISCLASSIFICATION: PLANETARY GEOLOGY}

Although there is as yet no standard definition of planetary geology (sometimes also known as astrogeology), it may be described as the study, on a wide variety of scales, of the origin, evolution and distribution of the matter condensed in the universe in the form of planets, satellites, asteroids, comets and particles of different sizes and genesis. It involves the incorporation of results from spacecraft data analyses, laboratory simulations of planetary processes, and field studies of features on Earth analogous to extraterrestrial features. ${ }^{3}$

Numerous wide-spectrum geological journals deal with planetary geology, for example Geology, Episodes and Geotimes. In a detailed search in the main Thomson Scientific Web of 
Science database for planetary geology-related papers published in the period 1978-2007, more than seventy-two per cent of record counts including the term 'planetary geology' corresponded to the five JCR (Journal Citation Reports) subject categories Geosciences, Multidisciplinary, Geology, Geochemistry, and Geophysics. Planetary geologists also often publish in certain astronomical/astrophysical journals - Icarus, for example - and the JCR category of Astronomy and Astrophysics accounted for a further sixteen per cent of record counts. Further, in the same search, ten journals belonging to the first five categories were the most used for publication in this thematic area, all with more than a thousand citations. ${ }^{4}$ Thus, the majority of studies and research topics coming under the planetary geology heading fall within UNESCO field 25, 'Earth and Space Sciences', and geologists and geoscientists are the main researchers involved in these kinds of activities.

Whilst the spread of work in planetary geology across the fields of earth and space sciences, with a smaller proportion in astronomy and astrophysics, is accurately reflected in the journals of the Web of Science database, in the UNESCO system by contrast it is altogether unacknowledged. Here, curiously, planetary geology appears only under the heading of Astronomy and Astrophysics, with the code 2104.04, whereas it does not appear in the field of Earth and Space Sciences (two-digit code 25) at all. This obliges both geologists and geoscientists working in planetary geology either to class their research within the field of Astronomy and Astrophysics, or alternatively within the less than helpful 'Other' category (code 2512.99) under the Earth and Space Sciences umbrella. In short, the standard UNESCO classification does not match the current reality of planetary geology.

The correction of this misclassification of planetary geology within the UNESCO system would, we propose, be an extremely simple matter. It would be sufficient to make a minor adjustment in the UNESCO standard nomenclature to group planetary geology within the Earth and Space Sciences field with the code 2512.04. Its present coding under the heading of Astronomy and Astrophysics (at 2104.04) could of course remain as it is, and then cross-references between the two code locations could be implemented, similar to those which already exist in many other fields. These straightforward refinements would avoid the structural discrimination built into the classification system against geologists and other geoscientists working in planetary geology, and would reflect the practical situation based on scientific collaborations and equitable relationships.

\section{A NOTABLE ABSENCE: ASTROBIOLOGY}

A second problem with the UNESCO standard system is perfectly represented by astrobiology and its conspicuous absence from the current classification. Astrobiology is an outstanding example of the development of interdisciplinary scientific studies over the last quarter-century. ${ }^{5}$ There are now university courses and seminars, postdoctoral grants, faculty positions, scientific institutions, prestigious books and ISI-rated journals (e.g. Astrobiology) connected with astrobiological research. The term 'astrobiology' dates back probably to 1941, when it was used by L. J. Lafleur as the title of a paper; ${ }^{6}$ it was subsequently used in 1953 in a book by the Russian astrophysicist G. A. Tikhov. ${ }^{7}$ However, fifty-six years had to pass from the term's first appearance before the publication in a 
Science Citation Index journal of an article in which it was mentioned. ${ }^{8}$ As it happens, the subject of that paper, the relationship between 'exobiology' and 'astrobiology', is extremely pertinent to an understanding of the problem of this subject's absence from the standard UNESCO classification. ${ }^{?}$

'Exobiology' is a term coined in 1960 to define a field of study that concerns itself with extraterrestial biology. Although some authors have argued for the equivalence of the two terms, the fact is that astrobiology was born as an attempt to go beyond biology and biochemistry and include a much broader range of disciplinary approaches. Astrobiology is a new word for a new paradigm. NASA recognised the possibility of looking into the origins of terrestrial life by conducting laboratory experiments and modelling bio- and geochemistry, subsuming exobiology, to reconstruct the events that led to biogenesis on the Earth from a cosmic perspective. ${ }^{10}$ Astrobiology is thus the study of the origins, evolution, distribution and future of life in the universe. It encompasses exobiology; the formation of elements, stars, planets and organic molecules; the initiation of replicating organisms; biogeological links in different terrestrial settings (analogues) and biological evolution; gravitational biology; and human exploration. Fundamentally, it tries to address three basic questions: How did life begin and evolve? Does life exist elsewhere in the universe? What is the future of life on Earth and beyond? ${ }^{11}$

The first appearance of the term 'astrobiology' in the ISI scientific databases was in 1997, and today, ten years later, there are around four hundred records of articles incorporating the term astrobiology, as against 229 records for exobiology. But whereas 'exobiology' appears in the UNESCO system as a subdiscipline of the Earth and Space Sciences field, with the code 2512.01, astrobiology remains absent. Thus, there is a clear need for astrobiology to be added to the standard UNESCO classification system. However, given astrobiology's highly interdisciplinary nature, the question would be where, and how, should it be reflected in the nomenclature? As mentioned above, UNESCO includes exobiology as a subdiscipline within the discipline of Space Sciences (code 2512). The results obtained after a bibliographic search for astrobiology, similar to the one described above for planetary geology, show that publications on this subject are split principally across three JCR categories - Astronomy and Astrophysics (41\%), Geosciences/Multidisciplinary (20\%) and Biology $(13 \%)$ - with papers in no other category reaching the ten per cent mark. Thus, a reasonable correction of the UNESCO system could be (1) to include astrobiology as a standalone discipline under the Astronomy and Astrophysics heading, say with code 2107, and (2) to add a suitable cross-reference within subdiscipline 2512.01 (Exobiology).

The United Nations, through its numerous commissions, committees and other agencies, contributes significantly towards moulding the current state and possible future evolution of scientific and technical knowledge on a global scale, as well as developing knowledge transfer mechanisms for development. ${ }^{12}$ In particular, UNESCO has a vital role as a clearing-house for the dissemination and sharing of information and knowledge, and as a think-tank and standard-bearer to forge universal agreements on emerging issues. The success and significance of its standard nomenclature for fields of science and technology provides clear evidence of this. Thus, the UNESCO codes are something of crucial 
importance: they are international quality indicators which reflect the ever changing panorama of the scientific 'tree of knowledge', independent from distorted views and biases based on particular interests, from the economic imperatives of the more lucrative applications of scientific and technical knowledge, and from the ups and downs of the research budgets associated with them. But if its classification system is to retain that status, UNESCO should also be alert to the innate dynamism and conceptual evolution of fields of knowledge and of scientific disciplines and subdisciplines, and so be always open to the incorporation of new and emerging areas of scientific endeavour.

\section{ACKNOWLEDGEMENT}

The authors would like to thank Howard Cattermole for valuable comments which greatly improved their manuscript.

\section{NOTES}

1. 'Problems encountered in the development of a standard international methodology of science statistics', UNESCO/CS/0666.SS-80/5, 1966.

2. 'Proposed International Standard Nomenclature for Fields of Science and Technology', UNESCO/NS/ ROU/257 rev.1, 1988.

3. Drawn from the homepage of the Planetary Geology Group at the Arizona State University (http://europa.la.asu.edu), this is in our opinion probably the best available characterisation of the field. Arizona State University is one of the most significant institutions in the world in this area, and the place where Eugene Shoemaker first coined the term 'astrogeology', in 1961. The Arizona definition is in broad agreement with the scientific guidelines of the USGS Astrogeology Research Program (http:// astrogeology.usgs.gov).

4. The journals in question are (with number of citations in brackets): Journal of Geophysical Research (all parts) (102 065), Geophysical Research Letters (30 488), Geocbimica Cosmocbimica Acta (28 126), Earth and Planetary Science Letters (20 325), Physics of the Earth and Planetary Interiors (3967), Advanced Space Research (3830), Journal of Atmospheric and Solar-Terrestrial Physics (3081), Meteoritics and Planetary Science (2436), Annual Review of Earth and Planetary Science (1973), Global and Planetary Change (1735). The search period was January 1978-January 2007.

5. C. S. Cockell: 'Astrobiology - a new opportunity for interdisciplinary thinking', Space Policy, 2002, 18, 263-266; B. S. Blumberg: 'The NASA Astrobiology Institute: early history and organization', Astrobiology, 2003, 3, 463-470; D. Morrison: 'The NASA Astrobiology Program', Astrobiology, 2001, 1, 3-13.

6. L. J. Lafleur: 'Astrobiology', Astronomical Society of the Pacific Leaflets, 1941, (143), 333.

7. G. A. Tikhov: Astrobiology; 1953, Moscow, Molodaya Gvardia Publishing House (in Russian). See also D. Briot, J. Schneider and L. Arnold: 'G. A. Tikhov and the beginnings of astrobiology', in Extrasolar Planets: Today and Tomorrow, (ed. J.-P. Beaulieu, A. Lecavelier des Etangs and C. Terquem), 219; 2004, San Francisco, CA, Astronomical Society of the Pacific.

8. G. A. Soffen: 'Astrobiology from exobiology: Viking and the current Mars probes', Acta Astronautica, 1997, 41, 609-611.

9. For an excellent summary of the terminological and conceptual confusion between 'astrobiology' and 'exobiology', see C. S. Cockell: “'Astrobiology” and the ethics of new science', Interdisciplinary Science Reviews, 2001, 26, 90-96.

10. See http://astrobiology.arc.nasa.gov/roadmap.

11. See, for example, K. W. Plaxco and M. Gross: Astrobiology: A Brief Introduction; 2006, Baltimore, MD, Johns Hopkins University Press; I. Gilmour and M. A. Sephton (ed.): An Introduction to Astrobiology; 2004, Cambridge, Cambridge University Press; J. Lunine: Astrobiology: A Multi-Disciplinary Approach; 2004, San Francisco, CA, Benjamin Cummings; G. Horneck and C. Baumstark-Khan: Astrobiology: The Quest for the Conditions of Life; 2001, Berlin, Springer-Verlag. 
12. J. Martínez-Frías and M. Hamdi: 'UNCSTD: "geoperspective" of a multidisciplinary commission', Interdisciplinary Science Reviews, 2002, 27, 83-86.

Jesús Martínez-Frías (martinezfrias@mncn.csic.es) is Head of the Planetary Geology Laboratory of the Spanish Centro de Astrobiologia (CAB) and was Vice-Chair of the United Nations Commission on Science and Technology for Development (UNCSTD) from 2002 to 2004. David Hochberg (hochbergd@inta.es) is a theoretical physicist and a member of the Transdisciplinary Laboratory at CAB. 